\title{
Les biens meubles du château de Gaillon
}

\author{
Laure FAGNART
}

Les sources en témoignent : au début d® $\mathrm{xvI}^{\mathrm{e}}$ siècle, le château de Gaillon ne retient pas seulement l'attention en raison de son architecture ou de l'aménagement de ses parcs et jardins. La richesse des décors intérieurs participe tout autant à la réputation de la résidence de Georges I ${ }^{\text {er }}$ d'Amboise. Ainsi, Bonaventura Mosti écrit dans la lettre qu'il adresse, le 24 septembre 1508, au duc de Ferrare?

"Gaglione, illustrissimo signore, (e luogo de ragione de lo archiepiscopato de Roanno, quale era andato in ruina. Et per essere in luogo de grande piacere per ogni generatione de caza, il reverendissimo signor legato lo ha facto il piu bello et superbo luoco sia in tuta la Franza [.... . Li solari de le sale et camare posti ad oro variamente. Quaranta otto allogiamenti vi sono, tuti atapezati di tapezarie del reverendissimo signor legato. A le lectiere, appramenti richissimi, et quilli migliori erano in la corte de Milano, vi sono, cum qualche guina etc. [... ${ }^{1}$.»

Jacopo Probo d'Atri, Y'envoyé d'Isabelle d'Este, qui visite la demeure d'été des archevêques de Rouen, entre le 20 et le 26 mars 1510, est lui aussi émerveillé par les cheminées, les plafonds à caissons, peints et dorés, les fenêtres ornées de vertrières historiées, les menuiseries des portes, les étoffes et les tapisseries décorant murs et mobilier, ainsi que par la bibliothèque, dans laquelle sont conservés des tableaux de chevalet, ou par le cabinet, qui lui est adjacentet que l'Italien désigne comme un "vero studiolo» :

"[... In capo de la bella logia se retrova la sala principale, alla quale la grandeza con l'atleza corresponde, con cimenere grande et magnifice, silicata

1. «Gaillon, Monseigneur illustrissime, est un lieu de la juridiction de l'archevêché de Rouen, qui était tombé en ruine. Et, comme il est dans un lieu d'un grand agrément pour toute sorte de chasse, monseigneur révérendissime le légat y a fait le plus beau et le plus superbe lieu qu'il y ait dans toute la France [...]. Les plafonds des salles et des chambres sont dorés de diverses façons. Il y a 48 logements, tous tendus de tapisseries de monseigneur révérendissime le légat. Aux lits, les garnitures les plus riches, et il y a là les meilleures qui étaient à la cour de Milan, avec quelques ajouts, etc. [...]. " Modène, AS, Cancelleria ducale, Estero, Carteggio ambasciatori, Francia, 4, cette lettre est publiée par Sмiтн M.-H., "Rouen-Gaillon : témoignages italiens sur la Normandie de Georges d'Amboise ", Beck B., Bouet P., Étienne C., Letteron I. (dir.), L'architecture de la Renaissance en Normandie, Actes du colloque tenu à Cerisy-la-Salle (octobre 1998) et monographies des principaux édifices Renaissance, Caen, 2003, p. 49. La traduction française est empruntée à M.-H. Smith. 
de petra cocta et il celo suffittato alla italiana, messo ad oro con vaghi et richi lavori, et le fenestre de vedro historiate et le figure si belle et perfecte che non scio quale pictore le potesse megliorare, et le porte et fenestre de legno ad ogni canto lavorate in tutta bellezza. Tacio li panni richamati d'oro facti ad aco et tapezarie, et li baldachini che sono sopra la sedia regale, però che sonno di quella richeza et belleza che se possa imaginare : ma solamente de un picolo me pare de parlare como da cosa rara, cioè de un poriale lavorato d'oro ad aguchia, dove è un Marte a cavallo armato che se retrova haver rotto la lanza et lo troncone anchora gli resta in mano et li pezi in terra, de tanta vivacia et perfectione che a pena la natura lo potrai far meglio, et il simile il cavallo, che se diria fossero l'uno et l'altro vivi. Intorno sonno trophei, arme et spoglie da non poterseli apponere in parte alcuna. Pare che fosse fatto fare da Lorenzo di Medici per donar al duca de Milano, che ben se conosce essere stato presente da grande homo et degno de conservarse fra cose pretiose et degne. Seguita poi una ben proportionata camera col cielo d'oro a similitudine de quello de la sala, con le fenestre de vedro simile, coperta de vellutocverde con le arme del legato et il lecto de panni d'oro et con molti altri panni rachamati [...]. In l'altro quadro, cioè incontro alla prima porta, è la libraria con la volta de legno messa ad oro, con alcuni quadri de pictura facti de mano de boni maestri, et lì contigue ̀̀ un gabinetto, o vero studiolo, lavoratold'oro con gioie de bon mercato ma di grande vista e bellezza, dove sonno grande quantità di libri scritti a penna in carta bona et coperti de veluto et d'oro ${ }^{2}$.

Ces descriptions rendent bien compte du luxe qui caractérisait les décors intérieurs du château de Gaillon. Ces textes ne permettent toutefois pas d'identifier avec exactitude quels objets ét quelles œuvres d'art y ont été conservés. Fort heureusemént, pour ce faire, d'autres sources nous sont

$\overline{2 . ~ «[\ldots] ~ E n ~ t e ̂ t e ~ d e ~ l a ~ b e l l e ~ l o g g i a ~ s e ~ t r o u v e ~ l a ~ s a l l e ~ p r i n c i p a l e, ~ d o n t ~ l a ~ g r a n d e u r ~ c o r r e s p o n d ~ a ̀ ~ l a ~ h a u t e u r, ~}$ avec de grandes et magnifiques cheminées, cette salle est pavée de pierres cuites, et le plafond, doré et travaillé richement et délicatement, est à caissons, selon la mode italienne, et les fenêtres avec des verrières historiées et des figures si belles et si parfaites, que je ne sais pas quel peintre pourrait les améliorer, et les portes et les fenêtres en bois, de chaque côté, d'une grande beauté. Je passe sous silence les étoffes brodêes d'or, travaillées à l'aiguille, les tapisseries et les baldaquins qui sont au-dessus du trône, parce qu'ils sont d'une richesse et d'une beauté que l'on ne peut imaginer : mais il me semble seufement devoir parler d'une petite chose, comme d'une chose rare, c'est-à-dire d'un poriale (?), travaillé d'or et à l'aiguille, où il y a Mars à cheval armé mais dont la lance est rompue (un morceau lui reste dans la main tandis que d'autres sont par terre), d'une telle vivacité et d'une telle perfection que la nature à peine aurait pu mieux les montrer, et de la même façon pour le cheval, à tel point qu'on dirait qu'ils sont l'un et l'autre vivants. Autour, il y a des trophées, des armes et des dépouilles, qu'on ne sait plus où les mettre. Il paraît que la pièce a été réalisée à la demande de Laurent de Médicis pour l'offrir au duc de Milan qui est bien connu pour avoir été un grand homme digne de rester entre des choses précieuses et dignes. Suit ensuite une chambre bien proportionnée dont le plafond est doré, de façon identique à celui de la salle précédente, avec les mêmes fenêtres à verrières, les murs de la pièce sont recouverts de velours vert avec les armes du légat et le lit est recouvert d'étoffes dorées et d'autres brodées. [...]. De l'autre côté, c'est-à-dire au-devant de la première porte, se trouve la bibliothèque, avec un plafond de bois doré et quelques peintures réalisées de la main de bons maîtres, et, à côté, se trouve un cabinet, véritable studiolo, travaillé à l'or et orné de joyaux à bon marché, mais d'un grand effet et d'une grande beauté, où se trouve une grande quantité de livres écrits à la plume et couverts de velours et d'or. " WeIss R., "The Castle of Gaillon in 1509-1510 ", Journal of the Warburg and Courtauld Institutes, t. 16, 1953, p. 1-12, 351. Nous remercions S. d'Arconso et F. Kieffer qui ont relu notre traduction française. 
parvenues : il s'agit, d'une part, des comptes de dépenses de la construction de la demeure, publiés en 1850 par A. Deville, qui font état d'achats ou de paiements réalisés dans le domaine de la tapisserie, de la peinture ou du livre ${ }^{3}$, d'autre part, de quatre inventaires (et non trois, comme on le dit trop souvent).

Le premier est intitulé Inventaire du mobilier du cardinal d'Amboise I'r , de son manoir archiépiscopal de Rouen ${ }^{4}$. Bien qu'il porte sur le palais rouennais, ce document constitue une source précieuse pour restituer l'aménagement intérieur du château de Gaillon : en 1508, une paftie du mobilier a été déplacée de Rouen à Gaillon, afin de préparer la visite que Louis XII et Anne de Bretagne y effectuèrent en septembre-octobre 1508. Incomplet du début (la partie supérieure du premier feuillet manque ; la seconde moitié de ce même feuillet est barrée) et non daté, facté est antérieur à 1508.

Le deuxième inventaire, intitulé Inventaire general des meubles a Monseigneur Messire le Légat estans tans à la maison archiépiscopal de Rouen que en ses chastaulx de Gailhon et Vigny, faict le XXe jour de septembre, l'an mil cinq cens et huyt ${ }^{5}$, soit la veille de la visite royale à Gaillon ${ }^{6}$, répertorie les biens meubles conservés à Gaillon, mais aussi ceux gardés au palais archiépiscopal de Rouen et au château de Vigny. À n'en pas douter, cet inventaire fut rédigé pour prévenir d'éventuels vols de biens meubles lors du séjour de la cour au châteaư Un second récolement fut réalisé sur ce même document, peu aprés la mort du cardinal, en 1510, comme l'indique la mention ajoutée en-tête. "Pour Monseigneur de Fescamp [il s'agit d'Antoine Bohier, abbe de Fécamp de 1506 à 1515, exécuteur testamentaire de Georges I" ${ }^{\text {er }}$ d Amboise] grant inventere de feu Monseigneur le légat. " Dans cet inventaire se distinguent d'ailleurs bien deux strates d'écriture, la seconde (de 1510) s'ajoutant à la précédente (de 1508). De plus, un fait intéressant pour@g tre propos peut s'observer : les rédacteurs précisent si

3. Deville A., Comptes de dépenses de la construction du château de Gaillon publiés d'après les registres manuscrits des trésoriers du cardinal d'Amboise, Paris, 1850.

4. Conservé à Rouen, ADSM, G 867, l'inventaire est publié par Deville A., Comptes de dépenses, op. cit., p. 486-499.

5. Conservé à Rouen, ADSM, G 866, ce document est également édité par Deville A., Comptes de dépenses, op. cit., mais, dans cette publication, comme l'a bien montré L.-A. Jouen, une partie des inventaires de 1508 et de 1550 ont été inversés. Voir Jouen L.-A., Comptes, devis et inventaires du manoir archiépiscopal de Rouen publiés avec une introduction historique par Mgr Fuzet, Paris-Rouen, 1908, p. 554. À ce propos, voir aussi Laffitte M.-P., «La librairie de Georges d'Amboise à Gaillon », Fabrizio-Costa S., Le Goff J.-P. (dir.), Léonard de Vinci entre France et Italie. "Miroir profond et sombre ". Actes du colloque international de l'université de Caen, Caen, 1999, p. 261-273, spécialement p. 263.

6. Sur la visite royale au château de Gaillon, voir l'article de Pagazani X. dans ce même volume. Voir aussi Bardati F., "Napoli in Francia ? L'arco di Alfonso e i portali monumentali del primo Rinascimento francese ", I Tatti Studies. Essays in the Renaissance, t. 11, 2007, p. 115-145.

7. Un cas de figure analogue se présente au château de Fontaine-le-Bourg en 1517 : l'abbé de Fécamp Antoine Bohier fait dresser un inventaire en avril, peu avant la venue de François $\mathrm{I}^{\text {er }}$ dans les lieux ; un récolement a lieu sur le document en 1519. Voir Pagazani X., Demeures campagnardes de la petite et moyenne noblesse en Haute-Normandie (1450-1600), thèse de doctorat, université Paris-Sorbonne, t. 4, 2009, doc. 4, p. 106. Nous devons ces informations à X. Pagazani que nous remercions. 
les pièces remontent à des acquisitions du cardinal, avec l'annotation «feu Monsieur le légat».

Qualifié d'Inventoire faict par nous Jehan de Bérat et Michel le Bret, serviteurs de reverend père en Dieu monseigneur Georges d'Amboise, archevesque de Rouen, par son commandement et ordonnance, de tous les biens meubles et utancilles estans de présent au chasteau de Gaillon appartenant à monseigneur, en la présence de Bernardin Guére, cappytayne dudit Gaillon, desquels biens ledict Guére est chargé ainsy et par la forme et manière que sensuyt, le troisième inventaire - non publié par A. Deville en 1850 - est Clressé à partir du 13 novembre $1540^{8}$. Postérieur à la mort de Georges IedAmboise, cet acte est extrêmement intéressant : même s'il remonte à l'époque de Georges II d'Amboise, neveu de Georges Ir et son successeur sur le trône archiépiscopal de Rouen, ce document permet d'en connaître davantage sur les aménagements réalisés à Gaillon au début du Xvi ${ }^{\mathrm{e}}$ siècle, Georges II ayant hérité de la majeure partie des biens de son ongle. Dans ce document, on retrouve les mentions de "feu Monseigneur " Ou "feu Monseigneur le légat».

Le quatrième et dernier inventaire est sans aucun doute le plus complet. Il est intitulé Inventaire faicte par nous Jeban Torel, conseiller du roy en sa court de parlement à Rouen, appellé avec nous et prins pour nostre adjoinct maistre Guillaume Goddefroy, principalcommys au greffe civil de ladite court, des biens meubles appartenans à l'archevesque de Rouen, d'autant qu'il en a esté par nous trouvé au chasteau de Gaillon, deppendent dudit archevesché, en vertu de la commission decernée par le roy et à nous presentée, donnée à Sainct Germain en Laye, le vingt septieme jour d'aoust mil v' cinquante, en la présence de $m^{e}$ Jehan Bignes, soy disant secretaire de monseigneur le reverendissime cardinal de Vendosme, et de Bernardin Guere, par cy-devant cappitaine dudit chasteau, le premier jour d'aoust oudit an $M . v^{e}{ }^{e}$ cinquante ${ }^{9}$. Achevé le 31 août 1550, ce document est rédigé à la mort de Georges II d'Amboise.

Jusqu'à présent, les historiens ont surtout utilisé ces comptes et ces inventaires pour restituer la chronologie du chantier de Gaillon, rétablir la distribution intérieure de la demeure ou reconstituer la célèbre bibliothèque de Georges 1'er d'Amboise. En revanche, on les a rarement exploités pour décrire laménagement intérieur du château ${ }^{10}$. La raison en revient

8. Ce document est conservé à Paris, BnF, ms. fr. 26533, fos 300r-313r. On en trouve une transcription dans Liou E.Y.L., Cardinal Georges d'Amboise and the château de Gaillon at the Dawn of the French Renaissance, thèse de doctorat, The Pennsylvania State University, 1997, p. 286-296.

9. Conservé à Rouen, ADSM, G 868, cet acte est publié par Deville A., Comptes de dépenses, op. cit., p. 529-559.

10. L'aménagement intérieur du château de Gaillon est rapidement évoqué par CHIRol É., Un premier foyer de la Renaissance en France. Le château de Gaillon, Rouen, 1952, notamment aux p. 63-65 et par BARDati F., "Il bel palatio in forma di castello ". Gaillon tra "Flamboyant " e Rinascimento, Rome, 2009, notamment aux p. 72-75. Quant à l'étude des collections de Georges Ir d'Amboise, elle a été entreprise par Souchal G., "Le mécénat de la famille d'Amboise ", Bulletin de la Société des Antiquaires de l'Ouest et des musées de Poitiers, $4^{\mathrm{e}}$ sér., t. 13, 1976, p. 485-526, 567-612, spécialement p. 578-588. Voir aussi la synthèse proposée par Toscano G., « Le cardinal Georges d’Amboise 
sans doute au fait que leur analyse ne permet pas de répondre à toutes les questions, les actes enregistrant, sans le préciser explicitement, des objets et des œuvres d'art conservés à Gaillon mais aussi au palais archiépiscopal de Rouen et dans le château de Vigny. Par ailleurs, l'enquête se révèle, au final, frustrante. En effet, de très nombreuses pièces - la plupart en fait - ont disparu ou elles ne sont plus associées au mécénat du cardinal (la famille d'Amboise étant puissante et étendue, les acquisitions, comme les successions, ont été nombreuses et compliquées). Enfin, en raison de l'imprécision des sources, dans lesquelles les attributions sont exceptionnelles et les descriptions des sujets représentés laconiques, rares sont les mentions que l'on peut assurément lier à une ouvre d'art ou à un objet parvenu jusqu’à nous.

\section{Les étoffes}

Dans le château de Gaillon, comme danslla plupart des demeures royales, seigneuriales et bourgeoises du temps, on trouvait de très nombreuses étoffes, à savoir des tapisseries, des tapis, des damas, des toques, des taffetas, des carreaux, des camelots, des écarlates ${ }^{11} \ldots$ On le sait, l'attrait pour de telles pièces s'explique non seulement par le plaisir esthétique que procure leur examen mais aussi, et surtout, par les fonctions pratiques qu'elles revêtent dans l'aménagement đes demeures. Par exemple, lorsqu'elles sont tendues sur les murs, les tapisseries permettent de calfeutrer les salles et d'éviter ainsi les déperditions de chạleur. Parfois rehaussées de fils d'or, d'argent ou de soie, ces étoffes constituent des pièces coûteuses que l'on enregistre consciencieusement dans les inventaires. Le cardinal d'Amboise ne fait pas exception : avec la vaisselle, souvent d'or ou d'argent, et les objets orfévrés - précieux, eux aussi, en raison des matériaux utilisés pour leur confection -, (Tes pièces tissées constituent les biens meubles qui sont le plus souvent mentionnés dans les inventaires des cardinaux d'Amboise.

Les sujets quildécorent les étoffes, tels que les mentionnent les comptes et les inventaires évoqués plus haut, paraissent conformes aux habitudes du

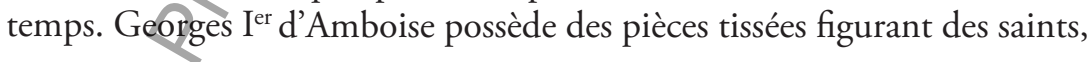

(1460-1510) collectionneur et bibliophile ", Lemerle F., Pauwels Y., Toscano G. (dir.), Les cardinaux de la Renaissance et la modernité artistique, Lille, 2009, p. 51-88, spécialement p. 55-61.

11. Jusqu'au xviII ${ }^{\mathrm{e}}$ siècle, rappelons que le mot "tapisserie " désigne tout textile destiné à couvrir meubles et murs; le tapis est une pièce d'étoffe dont on couvre une table ou un parquet; le damas est une étoffe précieuse qui présente des zones tantôt brillantes, tantôt mates ; la toque est une gaze d'or ou d'argent, fabriquée d'abord en Italie, qui est utilisée en ameublement ; le taffetas est un tissu servant pour les vêtements et l'ameublement des demeures; le carreau désigne un motif qui unit le pékin (rayures par armure, en chaîne) au bayadère (rayures en armure, en trame) ; le camelot désigne une étoffe à longs poils, comme une peluche, souvent de laine ; l'écarlate constitue une catégorie de draps prestigieux, tissés en laine fine d'Angleterre, souvent réservés aux personnages de haut rang. Voir Hardouin-Fugier É., Berthod B., Chavent-Fusaro M., Les étoffes. Dictionnaire historique, Paris, 1994, passim. 
parmi lesquels saint Georges, son saint patron ${ }^{12}$, saint Jean-Baptiste ${ }^{13}$ et saint Martin ${ }^{14}$. Les "tapisseries de verdure", par exemple ces " sept pieces de tapicerie de verdure à personnaiges bestes et oiseaulx, avec trois paviots ${ }^{15}$ ", sont également abondamment répertoriées, au même titre que les tapisseries de volerie ${ }^{16}$, celles de bergerie ${ }^{17}$, ou celles de "vignerons vendengeurs "... Georges d'Amboise détient encore des tapisseries dites "turques", comme ces "sept pieces de tappicerie de laine à la mode de Turquie " ou ces " huit granz tappiz de Turquie"18 ", ainsi que des productions "à la mode italienne ». En revanche, les sujets inspirés par la mythologie grecque et

12. " [...] une piece ou est l'imaige de sainct Georges faicte richement de broderye sur drap d'or [...] " dans l'inventaire de 1508 (Deville A., Comptes de dépenses, op. cit., p. 541); " [...] item le dossier ou est sainct Georges faict de riche broderye sur champ de velours bleu broche de fil d'or et la couverture du lict de semblable de velours bleu broché de fil d'or avec une bordure tout au tour de fort riche broderye [...]" dans l'inventaire de 1540 (Liou E.Y.L., Cardinal Georges d'Amboise, op. cit., p. 286). Le dossier est aussi cité dans l'inventaire de 1550, dans la "chambre de parement, prez ladite. salle haute " : "Ung dossier garni d'un sainct George de riche broderie sur champ de velours bleu broché de fil d'or avec une bordure à l'entour de fort riche broderie " (Deville A., Comptes de dépenses, op. cit., p. 534). Il s'agit sans doute de la "pièce où est l'image sainct Georges, fait sur drap d'or à brodeure ", qui est mentionnée dans l'inventaire du mobilier du manoir archiépiscopal de Rouen (ibid., p. 487). Rappelons que Deville (ibid., p. 534) transcrit par erreur "chambre duparc" au lieu de "chambre de parement". À ce propos, voir Bardati F., Chatenet M., Thomas É., "Le château de Georges Ir d'Amboise à Gaillon ", Beck B., Bouet P., Étienne C., Lettéron I. (dir.), L'architecture de la Renaissance, op. cit., p. 13-29, spécialement p. 19, n. 32.

13. "[...] ung ciel [soit un dais] de drap d'or ou est limaige de sainct Jehan Baptiste aulx armes de monseigneur [...]" cité dans l'inventaire de 1508 (Déville A.,Comptes de dépenses, op. cit., p. 541) ; "[...] item ung ciel de thoille d'or ou y a ung sainct Jehan de broderye aux armes de feu monseigneur le legat aux quattre coings avecque les pantes de mesmes, semez de rozes aux armes de monseigneur le legat et les franges de fil d'or et de soye " dans celui de 1540 (Liou E.Y.L., Cardinal Georges d'Amboise, op. cit., p. 286). Le ciel est également cité dans l'inventaire du mobilier du manoir archiépiscopal de Rouen (" [...] item, ung grand ciel de tolle d'or, garny de franges où est l'image et pourtraicture en bordeure de saint Jehan Baptiste avec les armes de monseigneur aux coings [...]. " Deville A., Comptes de dépenses, op. cit., p. 487) et dans l'inventaire de 1550, dans la "chambre de parement, prez ladite salle haute ": " ung ciel de toille d'or garny d'une ymaige de sainct Jehan de broderie aux armaries de feu monseigneur le legat aux quatre coings, avec les pentes de mesmes, semez de roses et les frenges de fil d'or et de soye " (ibid., p. 53

14. "[...] une aultre piece de sainct Martin faicte sur thoille d'or [...]" ainsi que le dit l'inventaire de 1508 (ibid., p. 541) ; " [...] item un piece pour la ruelle du lict de thoille d'or en laquelle y a au meillieu ung sainct Martin de broderye et les armes de feu monseigneur dont a esté desrobé environ quattre doigtz de large et ung aultre de long [...] " dans l'inventaire de 1540 (Liou E.Y.L., Cardinal Georges d'Amboise, op. cit., p. 286). Lá pièce est également mentionnée dans l'inventaire de 1550 , dans la "chambre de parement, prezladite salle haute" : " [...] une piece de toille d'or pour la ruelle du lict, au meilleur de laquelle est ung saint Martin de broderie garny aussi des armaries de feu monseigneur le legat, dont a esté derobbé envyron quatre doigts de large et ung autre de long". (Deville A., Comptes de dépenses, op. cit., p. 534).

15. D'après l'inventaire de 1508 (ibid., p. 544). Il s'agit de tapisseries où les feuillages tiennent la plus grande place. Des animaux et des personnages peuvent apparaître, mais habituellement ils ne sont pas impliqués dans une action concrète.

16. "[...] la tapisserie de volerie, contenant sept pieces, cing grandes et deux petites, aux armes de monseigneur sur lesdites cinq grandes pieces [...] " dans l'inventaire de 1508 (ibid., p. 547).

17. " [...] une tappisserie de verdure non bordée faicte à bergerie et à bestes, contenant six pieces aux armaries de feu monsieur le legat d'Amboise, servantes en la chambre du portail du milieu vers le jardin [...]" dans l'inventaire de 1550 (ibid., p. 520).

18. Dans l'inventaire du mobilier du manoir archiépiscopal de Rouen (ibid., p. 489, 591) et dans celui de 1540 (Liou E.Y.L., Cardinal Georges d'Amboise, op. cit., p. 290), lequel signale également une série, répertoriée dans une rubrique intitulée "tappis de turcquye ". 
romaine ou par l'histoire antique, que seigneurs et bourgeois commencent pourtant à posséder ${ }^{19}$, manquent à l'appel. Seule la tapisserie montrant Mars à cheval que Jacopo Probo d'Atri décrit longuement dans la " grande salle » du château de Gaillon, pourrait ici faire exception. Cette pièce ne peut toutefois pas être associée avec l'une des mentions enregistrées dans les inventaires que nous avons consultés.

Les sources nous apprennent encore que certaines salles de la résidence étaient décorées de somptueux ensembles tissés. Ainsi, la chambre de parade exécutée avec du velours vert, mentionnée par Jacopo Probo d'Atri en 1510, marqua les esprits. Le velours utilisé pour la confectionner avait été acheté à Gênes, puis enrichi à Tours, notamment par le brodeur Jean Galle, avant d'être envoyé à Gaillon, en 1508, en vue de la visite de Louis XII et d'Anne de Bretagne ${ }^{20}$. Les différentes pièces (tenture, garniture de lit et tapis) sont installées dans la salle dès lors dénommée "chambre de velours vert ", comme l'indiquent les inventaires de 1508 et de $1550^{21}$. C'est aussi le cas de la chambre de parade dorée qui est d'abord enregistrée à Rouen, au manoir archiépiscopal ${ }^{22}$, avant d'être envoyée à Gaillon, où elle est mentionnée en 1508 :

" [...] item, vingt-une piece dê tocque d'or qui servent à la chambre dorée du pavillon Nostre Dame, avec une coultre [couverture] de tafetas jaulne et ung ciel de damas jaulne ou il y aung soleil au millieu, avec la couchette de taffetas jaulne doublée de sarge vert sur lesquelles pieces y a quatre ymaiges de sainct Jehan et quatre armaries de monseigneur faictes de broderies ${ }^{23}$ ".

Enfin, conformément à la mode du temps, la plupart des étoffes du cardinal présentent ses armes, parfois ses devises. Quant aux mentions

19. Joubert F., Lefebure A., Bertrand P.-F., Histoire de la tapisserie. En Europe, du Moyen Âge à nos jours, Paris, 1995, p. 78.

20. Comptes de dépenses de la construction du château de Gaillon (Deville A., Comptes de dépenses, op. cit., p. 341-343).

21. En 1508, "en la chambre du bout après ladite chambre de parement", on trouvait donc "la tapisserie de veloux vert contenant neufz pieces, à chacune piece les armes de monseigneur faictes à rozies de drap d'or, et à chacune d'icelles quatre escriptiaulx de toille d'argent où sont escrips la devise de mondit seigneur, plus te ciel et pentes de semblables veloux et armes et devises que dessus, plus le lict en façon de lict de camp et les bastons couvers de veloux vert, plus troys rideaulx de taffetaz vert. Item, le banc et barre couvers de veloux vert, plus ung poisle de veloux vert ou sont les armes de mondit seigneur et devise dessusd. à frange de fil d'or et soye verte. Item, la couverture de veloux vert pour servir à la table. Item, deux carreaulx de veloux vert ou il y a à chacun deux houpes. Item, ung aultre carreau de mesmes sans houppes, plus une contre pointe de taffetaz vert doublée de mesme. Item, ung aultre tapis de mesme veloux doublé de toille verte servant au drescheur de ladite chanbre" (ibid., p. 543, 544). En 1550, " en la chambre apellée la chambre de velours vert, estans prez ladite chambre de parement" est répertoriée "une tapisserie de velours vert contenant neuf pieces en chacune desquelles sont les armaries de feu monseigneur le legat d'Amboise, faicte à restis de drap d'or et quatre escripteaulx de toille d'argent contenant la devise dudit feu sieur legat d'Amboise, et en la piece qui estoit au cheval du lict en a esté desrobé deux aulnes et demy-quart de long de la largeur d'un velours" (ibid., p. 534).

22. "[... [ item, XXI pieces de toque d'or qui servent à la premiere chambre du pavillon Nostre-Dame, avec une coultepoincte de taffetaz jaune et ung ciel de damas jaulne où y a ung soleil au millieu [...] " d'après l'inventaire du manoir archiépiscopal de Rouen (ibid., p. 488).

23. D'après l'inventaire de 1508 (ibid., p. 541). 
d'origine, elles sont rares ${ }^{24}$. De ce fait, il n'est pas aisé de retracer le cheminement des étoffes. En outre, comme l'histoire des pièces tissées en est coutumière, la plupart des biens répertoriés ont disparu (bon nombre d'entre eux sont d'ailleurs décrits, dès 1540 , en mauvais état de conservation ou en morceaux). Toutes, enfin, ne sont pas associées à Georges I ${ }^{\text {er }}$ d'Amboise. Il en va ainsi de la tapisserie emblématique sur fond de millefleurs, qui est conservée au musée départemental des Antiquités de Rouen (fig. 33 - cahier couleur). Habituellement considérée comme relevant du mécénat de Georges Ir d'Amboise, il convient plutôt dedassocier, comme $\mathrm{X}$. Pagazani nous l'a aimablement fait remarquer, à l'undes frères du cardinal, Jacques d'Amboise, abbé de Cluny : l'écu est bien celui de la famille d'Amboise ("palé d'or et de gueules de six pièces ", mais il est surmonté d'une crosse, et non de la croix archiépiscopale, qui, brdinairement, timbre les armes de Georges I $^{\text {er }}$.

\section{Georges I ${ }^{\text {er }}$ d'Amboise et les représentations de saint Jean-Baptiste}

On l'a déjà signalé, les inventaires ehregistrant les biens meubles des cardinaux d'Amboise consignent fréquemment des œuvres montrant saint Jean-Baptiste, témoignant ainsi de la dévotion de Georges I $^{\text {er }}$ pour ce saint. Parmi de très nombreux exemples, signatons " ung petit tableau d'or de Sainct Jehan Baptiste garny de pierres saphirs baletz et perles ${ }^{26}$ " ou " ung petit tableau où il y a sainct Jehan Baptiste et Saint Francoys, fait à l'esguille, où il y a de tunicâ in qua mortuus est sainct Francisce et saincte Clare ${ }^{27}$ ". Ces mentions sont même plus nombreuses que celles qui évoquent saint Georges ${ }^{28}$, saint

24. Quelques exceptions sont toutefoŕs à noter, comme ces pièces, provenant d'Angleterre, qui sont mentionnées dans l'inventaire de 1508 : «Troys cornetz et ung huchet [sorte de petit cor] donnés par le roy d'Angleterre, garnis desainctures de drap d'or " (ibid., p. 542).

25. Alors même qu'il n'est pas cardinal, Jacques d'Amboise fait parfois timbrer ses armes du "galero " (du chapeau cardinalice), quii, dans l'emblématique du temps, peut aussi désigner un prélat de haut rang. À ce propos, voir ljarticle de НАвLOT L. dans ce même volume. La tapisserie, sur laquelle sont figurés des bergers se désaltérant à une fontaine, perdue mais connue par une aquarelle de Roger de Gaignières, qui est conservée à Paris, BnF, département des estampes et des photographies, Rés. PCA18-FOL, fo 32r, doit sans doute aussi être associée à l'abbé de Cluny, même si on y retrouve, étrangement, la croix en pal : sur la bordure, les lettres I et $\mathrm{D}$ alternent avec des coquilles, ce qui constitue des emblèmes évocateurs du nom de Jacques d'Amboise et de son saint patron, saint Jacques.

26. D'après l'inventaire de 1508 (Deville A., Comptes de dépenses, op. cit., p. 501), il ne s'agit pas d'un tableau dans le sens moderne du terme mais d'une pièce d'orfevrerie, l'objet est d'ailleurs mentionné parmi la vaisselle d'or.

27. D’après l'inventaire du mobilier du manoir archiépiscopal de Rouen (ibid., p. 493). L'objet, enregistré dans la chapelle, est mentionné à Gaillon, dans l'inventaire de 1508, dans « la seconde chambre en portail sur le jardin " : "Ung petit tableau ou y a sainct Jehan Baptiste et sainct Françoys, faict à lesguille, ou il y a des rooés [sic] sainct Françoys et sainct Claire " (ibid., p. 538).

28. Crépin-Leblond T., «Fastes et décors liturgiques de la famille d'Amboise ", iD., TABuretDelahaye E. (dir.), L'art des frères d'Amboise. Les chapelles de l'hôtel de Cluny et du château de Gaillon. Catalogue de l'exposition tenue à Paris, musée national du Moyen Âge et à Écouen, musée national de la Renaissance. 3 octobre 2007-14 janvier 2008, Paris, 2007, p. 12. 
patron du cardinal, duquel il possédait une relique ${ }^{29}$. Comme l'a judicieusement proposé D.A. Brown, cette dévotion pourrait s'expliquer par le fait que saint Jean-Baptiste, alors considéré comme le meilleur intercesseur au moment du Jugement dernier, est aussi vénéré comme thaumaturge contre la goutte, la fièvre et la colique, maux qui accablaient le cardinal ${ }^{30}$.

Quoi qu'il en soit, la dévotion du cardinal pour saint Jean-Baptiste n'est pas seulement attestée par les inventaires. Cet attachement se discerne également dans des objets et des œuvres d'art. En témoigne le sceau d'archevêque de Rouen que s'est choisi Georges I ${ }^{\text {er }}$ (fig. 34). Amboise y est montré sous un dais gothique, agenouillé, priant la Vierge de pitié, avec saint Jean-Baptiste debout derrière lui, sur un fond semé de fleurs de lys ${ }^{31}$. De même, les deux moulages d'éléments de boiserie, provenant du château de Gaillon (peut-être dę tribune d'orgue de la chapelle), qui sont aujourd'hui conservés au musée du Louvre (RF 1265 et RF 1266), montrent le prélat de Rouen une fois avec saint Jean-Baptiste et la Vierge de pitié, une autre avegle saint prêchant face à des paysans ${ }^{32} \cdot \times 0$

Une commande, dont l'histoire a été reconstituée par G. Agosti et G. Toscano, manifeste aussi l'intérêt de Georgés I ${ }^{\text {er }}$ d'Amboise pour saint 2 ean-Baptiste 33 . Le 26 octobre 1499, tôrs de la conquête française du Milanais, Gemetto de Nessonis (Jamet de Nesson) soumet au marquis de

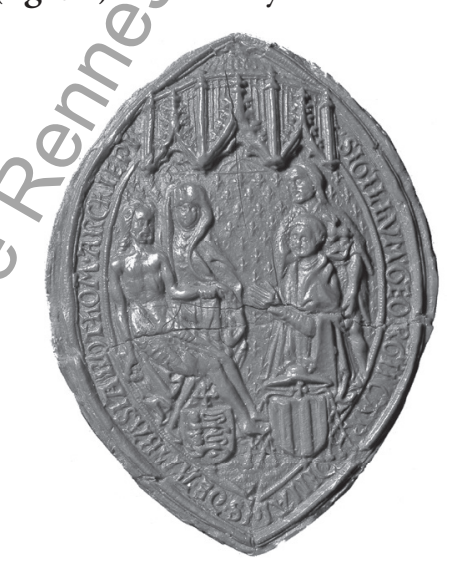

FIg. 34 - Sceau aux armes de Georges I ${ }^{\text {er }}$ d'Amboise, Rouen, musée départemental des Antiquités, inv. 1724.17, (C) cg $76-M D A$, F. DUGUE.

29. «[...] ung grant reliquaire de la jambe monsieur sainct Georges avec l'esperon rompeu qui est d'argent, avec le couvercle, pesant 21 mares 4 onces [...]" d'après l'inventaire du mobilier du manoir archiépiscopal de Rouren (Deville A., Comptes de dépenses, op. cit., p. 492).

30. Brown D.A., Andrea Solario, Milan, 1987, p. 161, 162.

31. Au bas du sceau, en cire rouge, figurent les armes de Normandie et celles de Georges ${ }^{\text {er }} \mathrm{d}^{\text {'Amboise, }}$ qui sont timbrées d'une croix en pal sous un chapeau cardinalice, avec des glands flanquant l'écu. Autour, on peut lire l'inscription suivante : "Sigillum Georgii Cardinalis de Ambasia Rothom Archiepi. "À propos du sceau, voir La Renaissance à Rouen. Catalogue de l'exposition de Rouen, musée des beaux-arts. 28 novembre 1980-28 février 1981, Rouen, 1980, p. 17, cat. 5.

32. Chirol É., Un premier foyer de la Renaissance, op. cit., p. 208-210 ; Gaborit J.-R. (dir.), musée du Louvre. Département des sculptures du Moyen Âge, de la Renaissance et des Temps modernes. Sculpture française, t. 2, Renaissance et Temps modernes, vol. 2, Goujon-Warin et anonymes, Paris, 1998, p. 616, 617 ; Crépin-Leblond T., "Le décor peint et sculpté de la chapelle de Gaillon », art. cit., p. 101.

33. Agosti G., "Su Mantegna, 2. (All'ingresso della maniera moderna) », Prospettiva, t. 72, 1993, p. 66-82, spécialement p. 67, repris dans ID., Su Mantegna, Milan, 2006, p. 110-111. Voir aussi Toscano G., "Per la fortuna di Mantegna in Francia ", Marinelli S., Marini P. (dir.), Mantegna e le arti a Verona 1450-1500. Catalogue de l'exposition tenue à Vérone, Palazzo della Gran Guardia. 16 septembre 2006-14 janvier 2007, Venise, 2006, p. 95-103, spécialement p. 95 ; ID., " Le cardinal Georges d'Amboise ", art. cit., p. 55-57. 
Mantoue, Gian Francesco II de Gonzaga, le désir de Georges d'Amboise d'acquérir une ouvre d'Andrea Mantegna, qualifié dans la lettre de " primo pictore del mondo ". Le tableau est destiné à orner la chapelle du palais archiépiscopal de Rouen. Le 31 octobre, le marquis de Mantoue répond favorablement à la requête du Français et lui demande de spécifier le sujet qu'il désire voir représenter dans le retable. Amboise opte pour une œuvre de format rectangulaire, qui le montrerait adorant saint Jean-Baptiste. Le cardinal promet aussi d'envoyer son " retracto dil naturale " et ses armes. Pour G. Agosti, le travail a été mené à son terme. C'escdu moins ce que laisse penser une annotation issue du Diaro de Marißo Sanudo et datée du $1^{\text {er }}$ juin 1500, qui témoigne d'un envoi au cardinat de Rouen ("Esso marchexe [...] manda una capella, val ducati 2000, a donar al cardinal $\left.\operatorname{Roam}^{34} »\right)$. Le chercheur propose en outre d'associer cette commande au dessin conservé à la Walker Art Gallery de Liverpool (fig. 35 - cahier couleur), dans lequel sont représentés un donateur et saint Jean-Baptiste, dans trois positions différentes. Pour G. Agosti, le dessin - dont la datation ne fait toutefois pas l'unanimité - a été exécuté durant les dernières années de l'activité de Mantegna : les solutions proposées pour figurer le saint sont marquées par un élan monumental et unle articulation des mouvements dans l'espace inconnus dans les œuvres dês années 1450, qui correspondent plutôt aux solutions privilégiées autour deş années $1500^{35}$.

Alors même que la reconstitution proposée parait judicieuse, on peut toutefois se demander si le tableau a bien eté conservé à Rouen, dans le palais archiépiscopal (en 1500, l'œuyre he peutyavoir été installée qu’à Rouen, à cette époque, les travaux débutant à peine à Gaillon). Comme l’a déjà souligné G. Toscano, le retable de Mantegna pourrait correspondre à " ung autre tableau comme sainct Jehan baptiste Notre Seigneur ", qui est répertorié avant 1508, dans l'inventaire du mobilier du manoir archiépiscopal de Rouen ${ }^{36}$. Le retable est enregistré dansla chapelle, ce qui constituerait un emplacement privilégié pour exposer une œuvre italienne, réalisée, de surcroît, par l'un des artistes les plus renømmés du temps, mais la mention n'est pas assez précise pour être assuré qửil s'agit bien du tableau de Mantegna, d'autant plus que, dans ces actes, on La vu, sont répertoriées de très nombreuses œuvres figurant saint Jean-Baptiste. On pourrait aussi s'étonner de ne pas voir apparaître, dans les comptes et les inventaires relatifs aux biens meubles des cardinaux d'Amboise, le nom d'Andrea Mantegna, un artiste que Georges d'Amboise appréciait tout spécialement et dont la fortune française, du moins au début du XVI ${ }^{\text {e }}$ siècle, est fortement liée au cardinal de Rouen.

34. Agosti G., «Su Mantegna, 2. ", art. cit., p. 111.

35. Agosti G., Thiebaut D. (dir.), Mantegna 1431-1506. Catalogue de l'exposition tenue à Paris, musée du Louvre. 26 septembre 2008-5 janvier 2009, Paris, 2008, p. 413 ; Marini G., "Andrea Mantegna. Tre studi per una figura di san Giovanni Battista ", Marinelli S., Marini P. (dir.), Mantegna e le arti a Verona, op. cit., p. 207-209, date quant à lui le dessin des environs de 1455-1460.

36. Deville A., Comptes de dépenses, op. cit., p. 494 ; Toscano G., "Le cardinal Georges d'Amboise », art. cit., p. 56. 


\section{La Cène d'après Léonard de Vinci}

En fait, dans les sources que nous avons consultées, les attributions sont rares. Ainsi, le nom d'un autre artiste - alors tout aussi célèbre qu'Andrea Mantegna - n'apparaît jamais dans les actes, alors même que l'une de ses compositions était conservée au château de Gaillon. Il s’agit de Léonard de Vinci dont une copie de la Cène de Santa Maria delle Grazie de Milan est répertoriée d'abord dans l'inventaire de 1540, dans une section consacrée aux tableaux ${ }^{37}$, puis, en 1550 , « en la grande gallerie » du château, à côté des " seize pulpittes de boys portez par ferreure contre la hucherie de ladite gallerie" qui présentaient les livres du cardinal ${ }^{38}$.

Alors même que le nom de l'Italien n'apparait jamais dans les inventaires, ces mentions se réfèrent sans aucun doufè̀ une version de la célèbre peinture murale de Léonard. Les sources nous apprennent en effet que cette Cène, peinte sur une toile, a été réalisée à Milan. Or, à cette époque, dans la capitale lombarde, l'œuvre du maitre toscan attise les convoitises, surtout auprès des Français qui la considèrent comme un ouvrage inestimable, au point d'en faire réaliser plusieurs copiesqu' ils ramèneront en France ${ }^{39}$. Les dimensions de la version de la Cène conservée à Gaillon étaient importantes puisqu'elles ont attiré l'attention des greffiers. L'indication en " grandz personnaiges " pourrait d'ailleưrs signifier que les apôtres étaient figurés grandeur nature, comme dans joriginal. En outre, comme l'indique la mention "feu monseigneur " K'ouvre a bien été exécutée avant la mort du prélat, soit avant mai 1510. Étant donné le nombre de fois que Georges I ${ }^{\text {er }}$ d'Amboise demeure à Milan et les fonctions qu'il occupe dans la ville, il a certainement visité le féfectoire du couvent Santa Maria delle Grazie ${ }^{40}$. Le cardinal a donc pûadmirer la peinture murale de Léonard et partager l'engouement des Franç̧ais pour le Cenacolo.

37. «[...] item, la Cenefaicte en toille en grandz personnaiges que feu monseigneur fist apporter de Millan [...] » d'après l'inventaire de 1540 (Liou E.Y.L., Cardinal Georges d'Amboise, op. cit., p. 292). Voir aussi Roman J. \&Note sur une ancienne copie de la Cène de Léonard de Vinci ", Réunion des Sociétés des beaux-arts des départements à la Sorbonne, 1883, p. 58-65.

38. "[...] une grande piece de toille paincte du pourtraict de la cene de Nostre-Seigneur [...]" d'après l'inventaire de 1550 (Deville A., Comptes de dépenses, op. cit., p. 536).

39. Voir Fagnart L., Léonard de Vinci en France. Collections et collectionneurs (XV'-XVII siècles), Rome, 2009, p. 151-173.

40. Le cardinal Georges Ir d'Amboise entre avec le roi à Milan, le 6 octobre 1499, et repart pour la France un mois plus tard, le 7 novembre. La seconde campagne du Milanais se termine le 17 avril 1500, avec l'entrée dans Milan et l'amende honorable des habitants. Jusqu'à la fin du mois de mai, le cardinal réorganise et réglemente le duché, puis, il laisse le gouvernement à son neveu, Charles II d'Amboise. Des séjours, non continus, le conduisent encore à Milan entre mai et octobre 1501, afin de préparer l'expédition de Naples. Durant l'été 1502, l'ecclésiastique est mentionné dans la capitale lombarde. Il passe sans doute par la ville en août 1503, avant de se rendre au conclave. En mars 1507, Amboise accompagne Louis XII au siège de Gênes. Ce voyage se termine le 24 mai 1507 par une entrée triomphale à Milan et par l'entrevue de Savone, en juin 1507, avec le roi d'Aragon. Amboise est encore cité en Lombardie en juillet 1509, après la victoire d'Agnadel et la prise de Brescia. 
Quoi qu'il en soit, malgré une abondante littérature qui lui a été consacrée, l'histoire de la Cène de Gaillon demeure, aujourd'hui encore, incertaine. Jusqu'il y a peu, l'une des hypothèses les plus séduisantes consistait à l'associer à la version de la Cène qui est aujourd'hui conservée au musée national de la Renaissance d'Écouen (fig. 36), une œuvre dont on sait qu'elle a été commandée par Gabriel Gouffier, chanoine puis doyen de Sens, en 1506, à Milan, puisque ses armes sont apposées sur les tréteaux de la table du repas et que l'acte de commande nous est parvenu ${ }^{41}$. Il a ainsi été proposé - nous de même dans d'autres pages ${ }^{42}$ - que Gabriel Gouffier, dont les liens avec le cardinal de Rouen sont attestés puisquil aurait obtenu sa charge de chanoine grâce à une permission spéciale du cardinal de Rouen ${ }^{43}$, ait pu servir d'intermédiaire lors de la commande d'une version de la Cène, destinée en fait au prélat. Georges d'Amboise aurait'également pu s'approprier la toile réalisée pour Gabriel Gouffier. Des recherches récentes, menées par A. Nassieu Maupas, viennent renouveler la question ${ }^{44}$. D'abord, contrairement à ce que l'on pensait jusqu'ators, Gabriel Gouffier n'est pas un membre de la célèbre dynastie Gouffier mais bien un homonyme d'origine auvergnate (ce qui explique la différence entre les armes apposées sur la Cène, "d'argent à la bande fuselée de sable ", et celles d'Artus Gouffier, par exemple, "d'or à trois jumelles de sable posées en fasce »). Par ailleurs, la Cène demeure en la possession de la famille(Gouffier durant tout le premier tiers du siècle au moins : en 1534, Guillaume Gouffier, chanoine de la cathédrale Notre-Dame de Paris, apparenté à Gabriel puisqu'il utilise les mêmes armes que le doyen de Sens, cherche à offrir l'œuvre aux chanoines de la cathédrale, en échange de messes prononcées pour le salut de son âme. En somme, il semble désormais difficile d'admettre que la copie commandée en 1506 par Gabriel Gouffier et celle acquise avant 1510 par Georges I ${ }^{\text {er }}$ d'Amboise constituent une seule et même œuvre. Il est plus raisonnable d'avancer que ces deux copies «françaises " du Cenacolo sont distinctes, laissant de ce fait ouverte la question de l'histoire ultérieure de la version mentionnée à Gaillon. Après 1550, en effet, on ne sait pas ce qu'est devenue cette Cène de Milan.

41. Sur la version de la Cène d'Écouen, voir Roman J., " Note sur une ancienne copie de la Cène ", art. cit., p. 58-65; Crépin-Leblond T., "L'origine de la copie de la Cène de Léonard par Marco d'Oggiono à Écouen ", Revue de l'art, t. 130, 2000, p. 60-62 ; FAGNART L., Léonard de Vinci en France, op. cit., p. 156-159. Notons que Gouffier séjourne aussi à Rome en 1506, sans doute pour porter devant le pape le compte-rendu d'un différend qui l'opposait à l'archevêque Tristan de Salazar, qui l'avait excommunié le 17 mars 1505. À ce sujet, voir Cailleaux D., La cathédrale en chantier. La construction du transept de Saint-Étienne de Sens d'après les comptes de la fabrique 1490-1517, Paris, 1999, p. 102.

42. Fagnart L., Léonard de Vinci en France, op. cit., p. 156-159.

43. En principe, pour devenir chanoine, il fallait, au préalable, être prêtre ou s'engager à le devenir. Or, en 1501, quand Gabriel Gouffier est reçu chanoine et archidiacre de Sens, il n'est que clerc. À ce propos, voir Cailleaux D., La Cathédrale en chantier, op. cit., p. 93, 94, 102.

44. Nassieu Maupas A., "Des mécènes méconnus : Gabriel Gouffier, doyen de Sens, et sa famille ", Elsig F. (dir.), Peindre en France à la Renaissance, t. 1, Les courants stylistiques au temps de Louis XII et de François I ${ }^{e r}$, Milan, 2011, p. 255-265. 


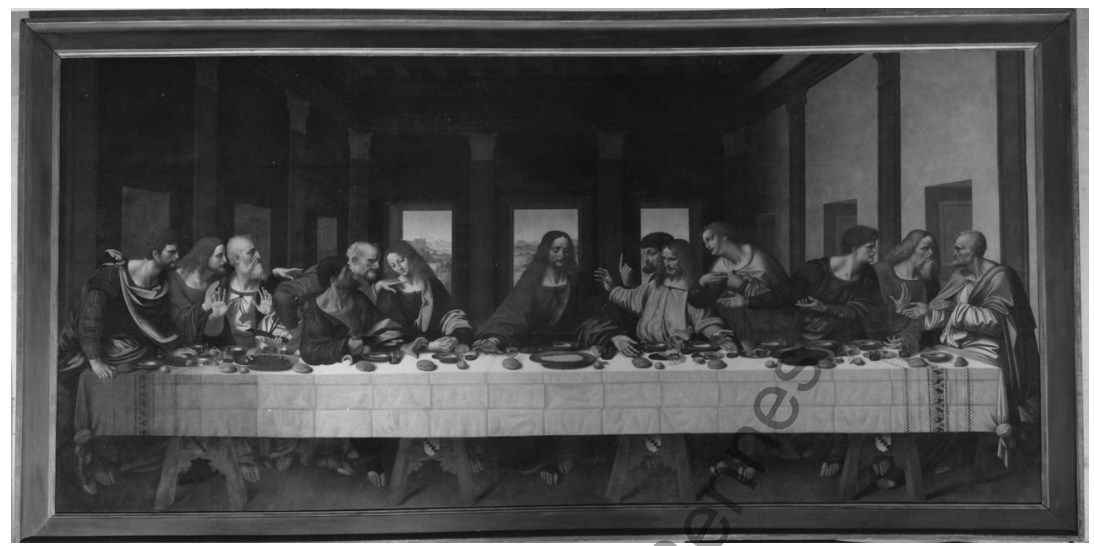

Fig. 36 - Marco D'OGGIONO, La Cène aux armes de Gabriel Gouffier, Écouen, musée national de la Renaissance, dépôt du musée du Louvre, inv. 781, (c) RMN-G. BLOT.

\section{Tableaux de Pérugin et d'Andrea Solario}

Ainsi, dans les sources qui nous inféressent, rares sont les noms d'artistes à être associés à des objets ou à des oeuvrees d'art en particulier. Deux exceptions sont toutefois à signaler : dans les inventaires des biens meubles des cardinaux d'Amboise sont répertoriés des tableaux de Pérugin et d'Andrea Solario.

À cette époque, comme la déjà signalé G. Toscano, Pérugin bénéficie d'une fameuse réputationen France ${ }^{45}$. Cette renommée s'est surtout développée sous le règne de Louis XII. En effet, dès la deuxième décennie $\mathrm{du} \mathrm{XVI}^{\mathrm{e}}$ siècle, les tableaux du maître ombrien ne sont plus à la mode, François I $^{\text {er }}$ appréciant plutôt la peinture contemporaine, à savoir celle de Léonard de Vinci et de Raphaël ; de ce fait, ce n'est probablement pas le premier roi Valois gui acquiert les œuvres de Pérugin qui sont conservées dans la collection royale française mais plutôt son prédécesseur ${ }^{46}$. Quoi qu'il en soit, Louis XII a probablement possédé deux, voire trois, tableaux de Pérugin, à sayoir le Saint Jérôme dans le désert, aujourd'hui conservé au musée des béaux-arts de Caen ${ }^{47}$, un Saint Sébastien, qui est perdu ${ }^{48}$, et

45. Toscano G., "Et Pérusin, qui si bien couleurs mesle : à propos de la fortune de Pérugin en France ", Ribemont F., Toscano G., Bret J. (dir.), Le Pérugin. La Vierge à l'Enfant entre saint Jérôme et saint Augustin, Paris, 1996, p. 13-21, 38-39.

46. Cox-Rearick J., Chefs-d'ouvre de la Renaissance. La collection de François I'r, Paris, 1995, p. 175.

47. On ne sait rien des circonstances de la réalisation de ce Saint Jérôme dans le désert dont la datation est à situer dans les dernières années du Xv ${ }^{\mathrm{e}}$ siècle. ADHEMAR J., "Une galerie de portraits italiens à Amboise en 1500 ", G.B.-A., t. 86, 1975, p. 99-104, spécialement p. 104, avait suggéré un rapprochement avec une œuvre qui est citée dans l'un des inventaires répertoriant les biens meubles d'Anne de Bretagne, mais la mention ("Ung tableau de Saint Jérosme paint») pourrait coïncider avec toute autre peinture montrant ce saint. Quoi qu’il en soit, l'œuvre est répertoriée à Fontainebleau, dans le cabinet des peintures du château, d'abord par Cassiano dal Pozzo en 1625 ("Un S. Girolamo col leone, et un paesetto ") puis par le père Dan en 1642 ("Un Sainct Hierème à genoux devant un crucifix »). Voir Cox-Rearick J., Chefs-d'cuvre de la Renaissance, op. cit., p. 175-177 ; Garibaldi V., 
peut-être aussi une Cléopâtre, qui a, elle aussi, disparu ${ }^{49}$. La renommée du peintre en France repose en outre sur la présence, dans les collections de Claude Gouffier, d'une Pietà de l'artiste (aujourd'hui à la National Gallery of Ireland de Dublin), qui a longtemps été conservée au château d'Oiron ${ }^{50}$.

Georges I ${ }^{\text {er }}$ d'Amboise aurait-il lui aussi possédé un tableau de Pérugin ? C'est probable puisque l'inventaire, rédigé avant 1508, du mobilier conservé dans le manoir archiépiscopal de Rouen enregistre « ung beau tableau où il y a une Nostre Dame de pitié paincte à plate painture de la main de Perusin ${ }^{51}$ ». En 1508, la peinture est citée à Gaillon, dans "la seconde chambre en portail sur le jardin", parmi d'autres Ceuvres, "le tableau de Perusin ou est la deposicion de Jesus-Christ avec plusieurs personnages ${ }^{52}$ ". Probablement envoyée à Gaillon, afin de décorer le château en vue de la visite de Louis XII et d'Anne de Bretagne, elle n'y est plus répertoriée par la suite, ni dans l'inventaire de 1540, ni dans lacte de 1550. La peinture de Pérugin a-t-elle été précocement détruite? A-t-elle été envoyée dans une autre demeure fréquentée par les cardinaux d'Amboise ? Dans l'état actuel des connaissances, ces questions restent sans réponse. Quoi qu’il en soit, l'œuvre ne nous est pas parvenue, à moins qu'il ne convienne de l'identifier avec un tableau d'Andrea Solario.

Mancini F. F. (dir.), Perugino. Il divin pittore. Catalogue de lexposition de Pérouse, Galleria nazionale dell'Umbria, Milan, 2004, p. 320, 321

48. Dans un contexte suggérant une date autour de 1500, Giorgio Vasari affirme qu'un Saint Sébastien a été commandé à Pérugin par le florentin Bernardino de’ Rossi, pour être envoyé en France ("Gli fu allogato da Bernardino de' Rossi, citadin fiorentino, un S. Sebastiano per mandarlo in Francia; e furono d'accordo del prezzo in cento scudi d'oro; la quale opera fu venduta da Bernardino al re di Francia quatrocento ducati d'oro "). Une miniature des Grandes Heures d'Anne de Bretagne de Jean Bourdichon montre saint Sébastien, debout dans un paysage, dans une position identique à celle visible dans d'autres ouvres de Pérugin qui figurent ce personnage (par exemple dans celle aujourd'hui conservée au musée du Louvre), ce qui peut attester du passage d'un Saint Sébastien debout de Pérugin dans lacollection royale française, même si son histoire en France reste imprécise. Voir Cox-Rearick J., Chefs-d'ouvre de la Renaissance, op. cit., p. 177, 178.

49. On sait peu de chose surPhistoire de ce tableau. En 1642, dans son Trésor des merveilles de la maison royale de Fontainebledú, le père Dan le répertorie dans le cabinet des peintures du château ( Le second est une Cléopatrê"), tout comme d'Estrechy, en 1692, dans son inventaire des tableaux restés à Fontainebleau après le déménagement de l'essentiel de la collection royale à Versailles ("Une Cléopâtre, sur bois, de Pierre Pérugin"). Un mauvais état de conservation ou un changement de goût pourraient expliquer pourquoi l'œuvre ne fut pas transférée dans la résidence de Louis XIV. Voir ibid., p. 178.

50. Daté d'entre 1493 et le début des années 1500, le tableau présentait les armes de Claude Gouffier, et de son épouse, Jacqueline de La Trémoille, avant qu'une restauration brutale ne les efface. L'étude de l'emblématique permet de supposer que les armes ont été apposées entre 1533, quand Claude reçoit le collier de l'Ordre de Saint-Michel, et 1545, quand son épouse décède. En 1683, la Pietà est mentionnée dans l'inventaire de la collection du duc et de la duchesse de La Feuillade, avant de passer dans la collection du duc d'Orléans au XviII ${ }^{\mathrm{e}}$ siècle. Voir MesLaY O., "Les collections de peinture des ducs de Roannez ", Crépin-Leblond T. (dir.), Les trésors du Grand Écuyer. Claude Gouffier, collectionneur et mécène à la Renaissance. Catalogue de l'exposition tenue à Écouen, musée national de la Renaissance, Paris, 1994, p. 85, 86 ; Toscano G., "Et Pérusin, qui si bien couleurs mesle", art. cit., p. 14.

51. Deville A., Comptes de dépenses, op. cit., p. 487.

52. Ibid., p. 540. 
De fait, certains ont associé la peinture de Pérugin (décrite donc comme une "Vierge de pitié" puis comme une "Déposition") avec la Déploration sur le Christ mort d'Andrea Solario, aujourd'hui conservé au musée du Louvre (fig. 37 - cahier couleur) ${ }^{53}$. Alors même que ce retable n'est jamais cité dans les comptes et les inventaires répertoriant les biens meubles des cardinaux d'Amboise, il est lié au mécénat de Georges I I ${ }^{\text {er }}$ En effet, il a été exécuté lors du séjour en France du peintre italien - venu à Gaillon, rappelons-le, afin de décorer la chapelle haute du château -, soit entre 1507 et 1510. De plus, à l'origine, il portait les armes du prélat de Rounen ${ }^{54}$. Chef-d'œuvre de Solario, bien connu des chercheurs, notamment parce qu'il présente l'un des premiers échos de la Vierge aux rochers de Léonard de Vinci ${ }^{55}$, le retable n'a toutefois pas encore livré tous ses secrets. Son histoire ancienne notamment demeure incertaine. À une date indéterminée, le tableau de Solario quitte les collections des cardinaux d'Amboise et passe (directement ou par l'intermédiaire d'un autre propriétaire ?) chez Claude de Longwy, évêque de Langres (à partir de 1528) et cardinal de Givry (à partir de 1533). Amateur d'art ${ }^{56}$, Claude de Longwy recouvre les armes de Georges I ${ }^{\text {er }}$ d'Amboise de son propre écu ${ }^{57}$. Par la suite, le retable est conservé dans le château de Tanlay, dans l'Yonne, oủ il est mentionné depuis 1672. Entre temps, il serait passé chez Françojse, épouse de Philippe Chabot, comte de Charny, et héritière du cardinal de Givry dont le petit-fils épousera Anne de Coligny, dame de Tanlay, ce quí explique-la présence de l'œuvre au château de Tanlay. En somme, on ne connait pas la destination originale de la peinture de Solario. Était-elle prévue pour Gaillon? Cela n'est pas certain. D'abord, elle n'est jamais, rappelons-le, mentionnée dans la résidence d'été des archevêques de Rouen. De plus, même si, on l'a vu, les rédacteurs des inventaires des biens des cardinaux d'Amboise sont peu enclins à procéder à des attributions, onjmagine mal qu'ils aient pu confondre une œuvre de Pérugin avec un tableau, signé et daté, de Solario, l'un des artistes italiens qui a expressémentservi le cardinal Georges I ${ }^{\text {er }}$ d'Amboise. Enfin, comme

53. Crépin-Leblond T., "Le décor peint et sculpté de la chapelle de Gaillon », art. cit., p. 105 notamment.

54. Peinte à tempera et à l'huile sur du chêne recouvert d'une toile de lin, l'œuvre est signée et datée mais les deux derniers chiffres de la date sont illisibles. Sur ce tableau de Solario, voir Beguin S. (dir.), Andrea Solario en France. Catalogue de l'exposition tenue à Paris, musée du Louvre. 15 novembre 1985-3 mars 1986, Paris, 1985, p. 72-81 ; Brown D.A., Andrea Solario, op. cit., p. 210, n 46 ; Bergeon S., Science et patience ou la restauration des peintures, Paris, 1990, p. 178-181 ; Beguin S., "Andrea Solario en France ", Fabrizio-Costa S., Le Goff J.-P. (dir.), Léonard de Vinci entre France et Italie, op. cit., p. 81-98, spécialement p. 93, 94.

55. FAgnaRT L., Léonard de Vinci en France, op. cit., p. 58, 59.

56. En juillet 1543, Claude de Longwy commande à Jean Cousin le père les cartons de la tenture dédiée à l'histoire de saint Mammès, destinée à orner la cathédrale de Langres dont il est l'évêque entre 1528 et 1561, quand il décède. Sur cette tenture, voir Auclair V., «L'iconographie humaniste d'un héros chrétien. Jean Cousin et la tenture de saint Mammès (1543) ", MichaUd-FréJAville F. (dir.), Tapisseries et broderies. Relectures des mythes antiques et iconographie chrétienne. Actes du colloque tenu à Angers, 4-6 octobre 2007, Angers, 2009, p. 118-128.

57. Bergeon S., Science et patience, op. cit., p. 178-181. 
l'indique T. Crépin-Leblond ${ }^{58}$, il est difficile de proposer une localisation à Gaillon. Dans la chapelle haute en tout cas, emplacement attendu pour exposer une peinture religieuse prestigieuse, italienne de surcroît, la place manque pour une œuvre de cette dimension, sauf si elle a été accrochée dans la tribune. Aussi, la Déploration sur le Christ mort de Solario - sans doute, si on admet qu'elle n'a jamais été accrochée à Gaillon, bien distincte de la Déposition de Pérugin - a plutôt été conservée dans un autre château du cardinal, peut-être à Vigny, dans lequel des œuvres d'art étaient gardées, comme l'indiquent les inventaires, ou à Chaumont-sur-Lôre, berceau de la famille d'Amboise, dans lequel certains biens, comme leślivres en français, ont été envoyés après le décès du cardinal, suivant ici une recommandation formulée dans son testament ${ }^{59}$.

L'hypothèse de l'existence de deux peintures différentes (une de Pérugin, une autre de Solario dont le parcours demeure, à chaque fois, incertain) se voit encore consolidée par le fait que, dans f'inventaire de 1508 , juste avant la Déposition de Pérugin, les rédacteurs qui enregistrent les biens des cardinaux d'Amboise signalent un autre tableau de Solario, distinguant ainsi clairement les deux artistes. Il s'agity d'une Nativité, décrite comme " ung beau tableau de la Nativité de Nostre Seigneur que a faict maistre André de Solario, peintre de monseigneur ${ }^{60} 8$. Cette œuvre a malheureusement disparu et son histoire demeure inconnue, puisqu'elle n'est pas citée dans les inventaires de 1540 et de 1550CAinsi, on ne sait pas si elle a été détruite ou si elle a quitté le château de Gaillon, peu après la mort du premier cardinal d'Amboise, rejoignant peut-êtré l'une des autres demeures de la famille. Quant aux autres ceuvres de Solario, considérées comme relevant du mécénat de Georges I ${ }^{e}$ d'Amboise, notamment en raison de leur provenance française et sur lesquelles il n'est pas le lieu de revenir ici ${ }^{61}$, elles ne sont jamais répertoriéés dans les comptes et les inventaires relatifs aux possessions des cardinaux d'Amboise.

\section{Autres ouvres peintes}

D'autres tábleaux de chevalet sont cités dans le château de Gaillon. On n'en connaît toutefois pratiquement rien : répertoriées sans nom d'auteur, sans mention de dimensions ou d'éléments significatifs qui permettraient de les reconnaître, ces œuvres ne sont pas associées à des productions actuelle-

58. CRÉpin-Leblond T., "Le décor peint et sculpté de la chapelle de Gaillon ", art. cit., p. 105.

59. "Item les livres en françoys seront portés à Chaumont. " Le testament de Georges Ir d'Amboise est conservé à Rouen, ADSM, G 3417. On en trouve une transcription dans Liou E.Y.L., Cardinal Georges d'Amboise, op. cit., p. 283-285. À propos des livres envoyés à Chaumont, voir l'article de LaffitTe M.-P. dans ce même volume.

60. Deville A., Comptes de dépenses, op. cit., p. 540.

61. À ce propos, voir Beguin S. (dir.), Andrea Solario en France, op. cit., passim; Brown D.A., Andrea Solario, op. cit., passim ; Beguin S., "Andrea Solario en France ", art. cit., p. 81-98 ; Fagnart L., Léonard de Vinci en France, op. cit., p. 207, 211, 212, 217, 218. 
ment encore conservées. Citons-les toutefois. Dans l'inventaire de 1508, les peintures sont signalées pour la plupart dans " la seconde chambre en portail sur le jardin ». On y trouve :

"[...] ung aultre tableau en boys ou est Notre Dame tenant son filz entre ses bras, ung aultre petit tableau ront ou est Nostre Dame tenant son filz et Jospeh, ung aultre petit tableau ou est figuré l'Anunciation, ung aultre petit tableau ou est Nostre Dame tenant son filz, ung aultre petit tableau ou est une mere Dieu fort noire, ung grant tableau de Nostre Dame de pitié et aultres ymages, ung aultre tableau comme sainct Jehan Baptiste baptize Nostre Seigneur, le grand tableau rond contenant Nostre Dame, son filz, ung petit sainct Jehan bourdé d'ung large bort d'or bruny, un moyen tableau carré ou est Jesus-Christ mort, deux petits tableaux carrés et couvers ou sont deux figures de sainct Jehan, ung tableau carré et couvers ou est ung Salvator mundi, la lignée des Roys de France faicte en ung rondeau de parchemin figuré par ung arbre començant Priamus le jeune et Anthenor et ung estuy de cuir noir ${ }^{62} "$.

L'histoire de ces tableaux, notamment celle de cette galerie de portraits des rois de France, que, à l'instar des souverains, les membres de la cour commencent eux aussi à collectionner, est inconnue. Les premiers proviennent de la chapelle du manoir archiépiscopal de Rouen ${ }^{63}$; ils ont probablement été envoyés à Gaillon peu avant 1508, quand le château est aménagé pour recevoir la visite déLouis-XII et d'Anne de Bretagne. Ces peintures ne sont toutefois pas répertoriées dans l'inventaire de 1540 , où l'on trouve mentionnés, semble-t-il, d'autres tableaux de chevalet. Ceux-ci, moins nombreux, apparaissent plus précieux, certains sont recouverts d'un volet, d'autres présentent de larges dimensions :

"[...] ung petit tableau carré fermant de boys couvert de verre où Notre Dame monte aux cieulx, item, ung tableau de cedre avec son couvercle auquel est Dieu porte croix et audit couvercle le sacrifice d'Abraham, item ung grand tableau on est sainct Georges 54 , item ung tableau ou est le trespassement de Nostre Dame, item ung aultre tableau ou Dieu prye au jardin d'oliviers ${ }^{65}$ ".

L'inventaire mentionne encore deux bas-reliefs ${ }^{66}$, qu'il est séduisant de rapprocher des médaillons qui ornaient la façade de la Grand' Maison 62. Deville A., Comptes de dépenses, op. cit., p. 539, 540.

63. "Ung autre tableau en boys où est une Nostre Dame tenant Nostre $S^{r}$ entre ses braz, ung autre petit tableau rond où est une Nostre Dame tenant son filz et Joseph, ung autre petit tableau rond où est figuré l'Annonciation, une autre Nostre Dame en petit tableau carré tenant son fils en ses bras, ung autre petit tableau ancien où y a une petite Nostre Dame fort noire, ung grand tableau de Nostre Dame de piété et autres ymages, ung autre tableau de Nostre Dame et saincte Helisabeth. "Ibid., p. 493, 494.

64. Selon T. Crépin-Leblond (CRÉpin-Leblond T., "Le décor peint et sculpté de la chapelle de Gaillon ", art. cit., p. 105), ce Saint Georges pourrait correspondre au "tableau d'excellente peinture de St Georges combattant le dragon pour la défense de la pucelle " mentionné dans la description que Godefroy donne en 1640 du château de Gaillon, au-dessus de la porte de la chapelle. Cette description est conservée à Paris, BnF, ms. fr. 20258.

65. Dans l'inventaire de 1540 (Liou E.Y.L., Cardinal Georges d'Amboise, op. cit., p. 292).

66. "Item deux medailles de marbre blanc faictes a visaige de femme augustynne" dans l'inventaire de 1540 (ibid.). 
du côté de la Seine et les portiques d'arcades de la cour d'honneur du château ${ }^{67}$. Enfin, dans l'inventaire de 1550, dans la " gallerie de la tappisserye ", on retrouve quelques-unes des œuvres déjà mentionnées dans l'acte de 1540 , à savoir :

«[...] deux medalles de marbre blanc à visage de femme Augustine, ung tableau carré de boys fermant couvet de voirre qui est una assomption de Nostre Dame, ung tableau de cedre avec son couvercle, ouquel est l'image de Nostre Seigneur portant croix, et oudit couvercle l'effigie du sacrifice d'Abraham, ung grand tableau auquel est l'effigie de sainct Georges, ung tableandu trepassement de Nostre-Dame, ung autre tableau ouquel est l'effigie Nostre Seigneur priant au jardin d'Olivet ${ }^{68} \%$.

Finalement, comme pour les tapisseries et les pièces tissées, les sujets des tableaux de chevalet répertoriés dans les inventaires des biens meubles des cardinaux d'Amboise sont traditionnels, l'iconographie demeurant fondamentalement religieuse : y sont surtout signalêes des peintures montrant la Vierge, accompagnée ou non de l'Enfant, đess saints, saint Jean-Baptiste en tête; quelques thèmes issus de l'Ancieñet du Nouveau Testament. En revanche, on ne trouve pas de tableaux mythologiques, de paysages ou de nus, dont la présence aurait pu, ên ce début du XVI ${ }^{\mathrm{e}}$ siècle, témoigner d'un goût au fait des nouveautés du temps, et qui se retrouveront en grand nombre dans les cabinets des curieux de la seconde moitié du XVI et du $\mathrm{XVII}^{\mathrm{e}}$ siècle. À l'exception de la galerie de portraits des rois de France, les portraits sont absents eux aussi

\section{5}

Peut-on conclure de ce survol de l'aménagement intérieur du château de Gaillon que Georges I ${ }^{\text {er }}$ d'Amboise fut un amateur avisé ? Les comptes et les inventaires qui hous sont parvenus n'autorisent pas une réponse unique. D'un côté, le prélat de Rouen semble surtout soucieux de luxe et de confort. En témoignent les nombreuses étoffes et tapisseries répertoriées dans les documents d'archives mais aussi la vaisselle d'or et d'argent, que nous n'avons pas evoquée. Cette impression se renforce encore au vu des sujets des œuvres d'art qui ont été conservées à Gaillon (mais aussi à Rouen et à Vigny) : religieux pour la plupart, ils ne mettent pas à l'honneur de nouvelles thématiques, d'inspiration antique notamment, qui seront tant appréciées dans les années qui viennent. De même, au vu des sources que nous avons consultées, le château de Gaillon ne semblait pas destiné à

67. Bresc-Bautier G., "Médailles et profils d'applique en marbre ", Pagazani X., Bresc-Bautier G., Hellas S. (dir.), Le château de Gaillon. Fastes de la Renaissance en Normandie. Catalogue de l'exposition tenue à Rouen, musée départemental des Antiquités. 27 février-12 mai 2008, Rouen, 2008, p. 13-27 ; Bardati F., "Il bel palatio ", op. cit., p. 93-101.

68. Deville A., Comptes de dépenses, op. cit., p. 532. 
conserver et à rassembler des sculptures antiques ${ }^{69}$ ou contemporaines (en dehors de celles relevant du décor architectural), mais aussi des médailles, des monnaies, des émaux, des pierres gravées, soit toutes ces " petites antiquités " que certains recherchent alors avidement. De l'autre, on le sait, Georges I ${ }^{\text {er }} \mathrm{d}^{\prime}$ Amboise a rassemblé une exceptionnelle bibliothèque. Par ailleurs, il s'est intéressé à l'architecture comme peu de ses contemporains, privilégiant les formes et les fonctions nouvelles, spécialement celles en vigueur en Italie. Il a aussi joué un rôle décisif dans la fortune française d'Andrea Mantegna et de ses œuvres, tout comme il a activement participé à la diffusion au Nord des Alpes du goût pour les productions italiennes, en se procurant notamment des compositions de Léonard de Vinci, Pérugin et Andrea Solario. En somme, le portrait de Georges I ${ }^{\text {er }} \mathrm{d}^{\text {'Amboise collec- }}$ tionneur doit être nuancé : lettré et mécène, certainement, collectionneur dans le sens où on l'entend au début du XVI sjiècle, peut-être pas.

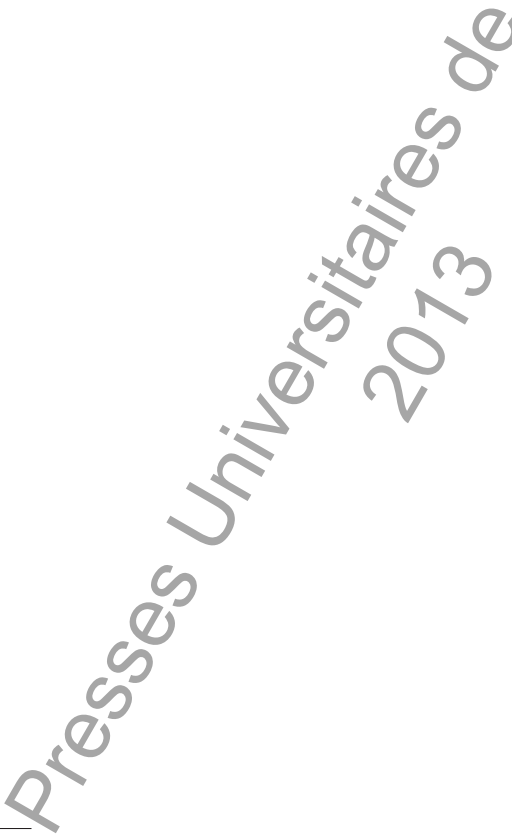

69. Le cardinal d'Amboise aurait toutefois cherché à acquérir un Bacchus comme en témoigne une série de lettres, conservées à l'AS de Modène et échangées, durant les mois de mars et d'avril 1505 , entre Alfonso d'Este, duc de Ferrare, et son ambassadeur à Milan, Girolamo Seregno. En effet, dans une lettre du $1^{\text {er }}$ avril 1505 , Alphonse d'Este demande à son ambassadeur d'acquérir un Bacchus, qui appartient alors à Antonio Maria Pallavicino, mais, le 17 avril, l'ambassadeur transmet au duc les regrets de Pallavicino, qui ne dispose plus du Bacchus étant donné qu'il l'a promis au cardinal Georges I $^{\text {er }}$ d'Amboise. Pendant longtemps, la critique a associé ces mentions à une peinture de Léonard de Vinci, alors même que le nom de l'Italien n'apparaît en fait jamais dans les lettres. Villata E., Leonardo da Vinci. I documenti e le testimonianze contemporanee, Milan, 1999, p. XVI-XVIII, en rééditant ces sources, en conclut judicieusement que l'on ne peut associer ces textes à une œuvre de Léonard et qu'il s'agit peut-être d'une sculpture antique. Quoi qu'il en soit, ce Bacchus n'est jamais évoqué à Gaillon, ni dans les comptes de dépenses de la construction du château, ni dans les inventaires des biens meubles des cardinaux de Rouen. 


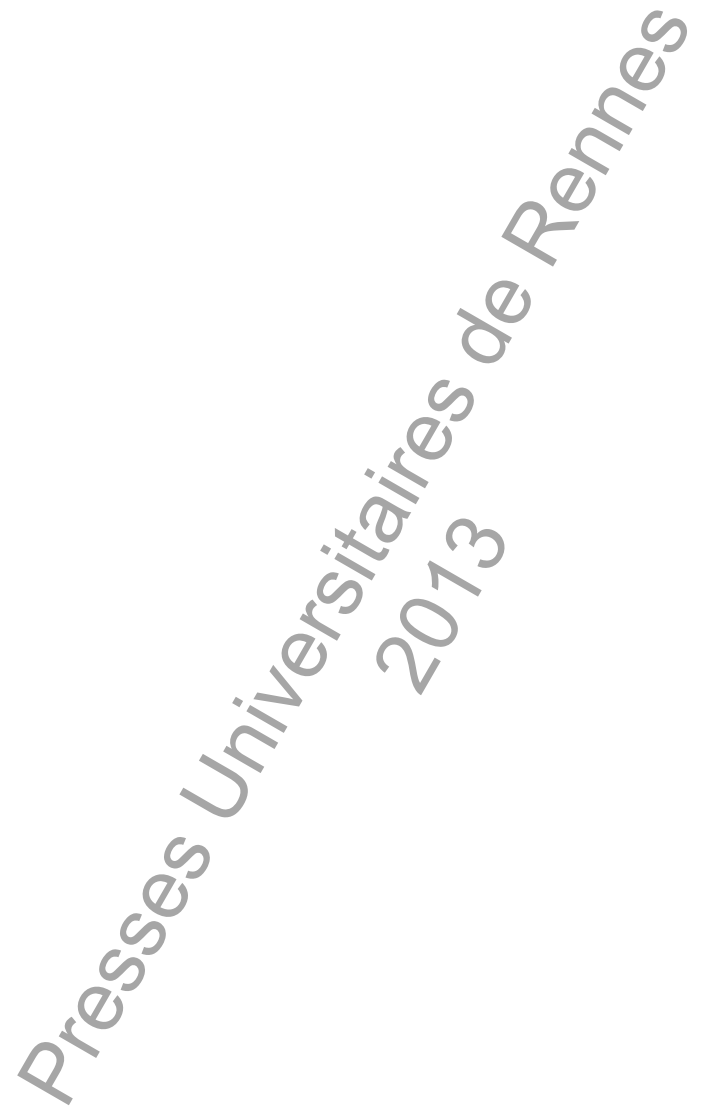

